Pacific Journal of Mathematics

MATRIX TRANSFORMATIONS INVOLVING SIMPLE 


\title{
MATRIX TRANSFORMATIONS INVOLVING SIMPLE SEQUENCE SPACES
}

\author{
ROY T. JACOB, JR.
}

\begin{abstract}
One property of spaces of analytic or entire sequences is that every bounded subset of each of them is contained in the normal hull of a single point of the space. In this paper sequence spaces having this property are studied and many characterizations of matrix transformations involving these spaces and their duals are shown to involve it. Several simple theorems about matrix transformations are proved, and many of the known theorems about matrix transformations on analytic and entire sequences are shown to be special cases of these general theorems.
\end{abstract}

A sequence space is a linear space each point of which is an infinite complex sequence. If $\lambda$ is a sequence space then $\lambda^{*}$, the dual (or $\alpha$-dual or Köthe dual) of $\lambda$, is the collection of all infinite complex sequences $y$ such that $\sum_{k=0}^{\infty}\left|x_{k} y_{k}\right|$ converges whenever $x$ is a point of $\lambda$.

A sequence space $\lambda$ is called perfect if $\lambda=\lambda^{* *}$. Clearly, the dual of every sequence space is perfect. Some examples of perfect sequence spaces are the space $\omega$ of all complex sequences, the space $\varphi$ of all complex sequences that do not have infinitely many nonzero terms, the space $l$ of all absolutely convergent complex sequences, and the space $m$ of all bounded complex sequences.

A sequence space $\lambda$ is said to be normal provided that whenever $y$ is a point of $\lambda$ and $x$ is a complex sequence such that $\left|x_{n}\right| \leqq\left|y_{n}\right|$ for every nonnegative integer $n$ then $x$ is also a point of $\lambda$. Every perfect space contains $\varphi$ and is normal.

For each sequence space $\lambda$ that contains $\varphi$, a dual system with $\lambda^{*}$ is formed using the bilinear functional

$$
Q(x, y)=\sum_{k=0}^{\infty} x_{k} y_{k}
$$

where $x$ is a point of $\lambda$ and $y$ is a point of $\lambda^{*}$. Under this duality, $\lambda$ is provided with its usual dual system topologies.

Theorems A and B are due essentially to Köthe and Toeplitz and Allen (see [12], Satz 1, p. 208, and [6], Theorem 6.2.II).

THEOREM A. Suppose each of $\lambda$ and $\mu$ is a sequence space and $\lambda$ contains $\varphi$ and is normal. A linear transformation from $\lambda$ to $\mu$ is a matrix transformation if and only if it is weakly continuous. 
Whenever each of $\lambda$ and $\mu$ is a sequence space then $(\lambda, \mu)$ denotes the set of all infinite matrices that transform $\lambda$ into $\mu$.

THEOREM B. Suppose each of $\lambda$ and $\mu$ is a sequence space, $\lambda$ contains $\varphi$ and is normal, and $M$ is an infinite matrix. Condition (1) implies condition (2):

(1) $M$ is in $(\lambda, \mu)$;

(2) $M^{\prime}$ (the transpose of $M$ ) is in $\left(\mu^{*}, \lambda^{*}\right)$.

Whenever $\mu$ is perfect, these two conditions are equivalent.

1. Simple Sequence Spaces. A point $y$ of a sequence space $\lambda$ is said to dominate a point set $X$ in $\lambda$ provided $\left|x_{n}\right| \leqq\left|y_{n}\right|$ whenever $n$ is a nonnegative integer and $x$ is a point of $X$. A sequence space is said to be simple whenever each bounded point set in the space is dominated by a point of the space.

THEOREM 1. If $\lambda$ is a simple sequence space that contains $\varphi$ then $\lambda^{* *}$ is simple.

Proof. Suppose $X$ is a bounded subset of $\lambda^{* *}$. Consider the set $Y$ to which $y$ belongs if and only if there exist a point $x$ in $X$ and a nonnegative integer $n$ such that $y$ is the sequence $\left(x_{0}, x_{1}, \cdots, x_{n}, 0,0, \cdots\right)$. The point set $Y$ is a bounded subset of $\lambda$, so it is dominated by some point of $\lambda$. This point also dominates $X$. Therefore, $\lambda^{* *}$ is simple.

THEOREM 2. If $\lambda$ is a simple space then a point sequence is weakly Cauchy in $\lambda$ if and only if it is bounded and coordinatewise convergent.

Proof. Let $y$ denote a point of $\lambda$ that dominates the bounded, coordinatewise convergent point sequence $\alpha$. For each point $u$ of $\lambda^{*}$,

$$
\sum_{k=0}^{\infty}\left|\alpha_{n k} u_{k}\right| \leqq \sum_{k=0}^{\infty}\left|y_{k} u_{k}\right|<\infty,
$$

so $\sum_{k=0}^{\infty} \alpha_{n k} u_{k}$ is uniformly convergent. It follows that the complex sequence $\left\{Q\left(\alpha_{n}, u\right)\right\}$ converges. Therefore, $\alpha$ is weakly Cauchy.

Since in every sequence space, every weakly Cauchy point sequence is bounded and coordinatewise convergent, the theorem is proved.

COROLlary 1. If $\lambda$ is a simple space then every bounded point sequence in $\lambda$ has a weakly Cauchy subsequence.

Indication of Proof. Corollary 1 can be proved by an easy but tedious diagonalization argument. 
Corollary 2. If $\lambda$ is a simple space, $X$ a bounded set in $\lambda$, and $x a$ weak limit point of $X$, then $x$ is a weak sequential limit point of $X$.

Proof. The set $X$ is bounded in $\omega$, and the point $x$ is a weak limit point in $\omega$ of $X$. Since the weak topology on $\omega$ is metrizable, there is a sequence of points from $X$ converging weakly in $\omega$ to $x$, i.e. converging coordinatewise to $x$. This sequence converges to $x$ in $\lambda$.

COROLlARY 3. If $\lambda$ is a simple space then the weak topology on $\lambda$ is metrizable on each bounded set in $\lambda$.

Proof. By Corollary 2 and its proof, the topology induced on a bounded set $X$ by the weak topology of $\lambda$ is identical with that induced on $X$ by the weak topology of $\omega$, which is metrizable.

THEOREM 3. Every normal simple space is weakly sequentially complete.

Proof. Suppose $\lambda$ is a normal simple space, $\alpha$ a weakly Cauchy point sequence in $\lambda, x$ the weak limit of $\alpha$ in $\lambda^{* *}$, and $y$ a point of $\lambda$ that dominates $\alpha$. Since $\left|\alpha_{j k}\right| \leqq\left|y_{k}\right|$ whenever each of $j$ and $k$ is a nonnegative integer and $x_{k}=\lim _{j} \alpha_{j k}$ for each nonnegative integer $k$, it is also true that $\left|x_{k}\right| \leqq\left|y_{k}\right|$ for each nonnegative integer $k$. Since $\lambda$ is normal, $x$ is a point of $\lambda$. Consequently, $\lambda$ is weakly sequentially complete.

Corollary 4. A simple space is perfect if and only if it contains $\varphi$ and is normal.

Indication of Proof. Corollary 4 follows from Theorem 3 and $30.5(3)$ of [11].

THEOREM 4. If $\lambda$ is a sequence space containing $\varphi$ whose dual is simple then the weakly convergent (Cauchy) sequences in $\lambda$ are the same as the strongly convergent (Cauchy) ones.

Proof. In a sequence space every strongly convergent (Cauchy) point sequence is obviously weakly convergent (Cauchy).

Suppose $\alpha$ is a point sequence converging weakly in $\lambda$ to 0 . Suppose further that $Y$ is a bounded subset of $\lambda^{*}$, that $y$ is a point of $\lambda^{*}$, every term of which is a nonnegative real number, that dominates $Y$, and that $\epsilon>0$. Let $\beta$ denote the point sequence in $\lambda^{* *}$ such that $\beta_{\jmath k}=\left|\alpha_{\jmath k}\right|$ whenever each of $j$ and $k$ is a nonnegative integer. The sequence $\beta$ converges weakly in $\lambda^{* *}$ to 0 ([10], Hilfssatz 1, p. 74), so there is a positive integer $n$ such that $Q(\beta, y)<\epsilon$ whenever $j$ is an integer greater 
than $n$. Thus, whenever $u$ is a point of $Y$ and $j$ is an integer greater than $n$,

$$
\begin{aligned}
\left|Q\left(\alpha_{j}, u\right)\right| & =\left|\sum_{k=0}^{\infty} \alpha_{j k} u_{k}\right| \\
& \leqq \sum_{k=0}^{\infty} \beta_{j k} y_{k} \\
& <\epsilon .
\end{aligned}
$$

Therefore, $\alpha$ converges strongly in $\lambda$ to 0 . It follows that if $\alpha$ is a point sequence converging weakly in $\lambda$ to the point $x$ then $\alpha$ also converges strongly in $\lambda$ to $x$.

If $\alpha$ is a weakly Cauchy point sequence in a (not necessarily perfect) sequence space $\lambda$ then $\alpha$ is weakly convergent in $\lambda^{* *}$. Since $\lambda^{* *}$ is simple (by Theorem 1), $\alpha$ is strongly convergent in $\lambda^{* *}$. Consequently, $\alpha$ is strongly Cauchy in $\lambda$.

Theorem 4 generalizes a number of theorems in the literature, including Theorem 15 of Lascarides [13].

2. Spaces of analytic sequences. If $0 \leqq r<\infty$, let $\mathscr{S}_{r}$ denote the collection of all complex sequences $x$ such that $\lim \sup _{n}\left|x_{n}\right|^{1 / n} \leqq$ $r$; and if $0<r \leqq \infty$, let $\mathscr{F}_{r}$ denote the collection of all complex sequences $x$ such that $\lim \sup _{n}\left|x_{n}\right|^{1 / n}<r$.

In [18] Toeplitz established many topological properties of these spaces. His results relevant to this paper are listed below:

THEOREM C. If $0 \leqq r<\infty$, then $\mathscr{S}_{r}$ and $\mathscr{J}_{1 / r}$ are dual to each other and, hence, are both perfect (where $1 / 0=\infty$ ).

THEOREM D. (1) If $0 \leqq r<\infty$, then $\mathscr{S}_{r}$ is simple;

(2) If $0<r \leqq \infty$, then $\mathscr{F}_{r}$ is simple.

The following statement is equivalent to (2) and often more useful:

$\left(2^{\prime}\right)$ If $X$ is a bounded point set in $\mathscr{F}_{r}$, then there exist numbers $t$ and $s$ such that $0<s<r$ and $\left|x_{n}\right| \leqq t s^{n}$ whenever $x$ is a point of $X$ and $n$ is a nonnegative integer.

\section{Matrix transformations involving simple sequ-} ence spaces. In this section several basic theorems about matrix transformations involving simple spaces are stated and most of the theorems about matrix transformations on $\mathscr{S}_{r}(0 \leqq r<\infty)$ found in the literature are shown to follow from these general ones.

It should be noted that this work is similar in spirit to parts of the papers of Bennett [1] and Snyder and Wilansky [17] in that a simple, 
possibly previously unnoticed, idea is used to unify a number of scattered results already in the literature. However, their results generally do not apply to the spaces studied here since perfect simple sequence spaces are not necessarily $F K$ spaces.

Our first theorem is an immediate consequence of the definitions:

THEOREM 5. Suppose $\lambda$ is a perfect simple space and $M$ is an infinite matrix. The following are equivalent:

(1) $\quad M$ is in $\left(\lambda^{*}, m\right)$;

(2) $M^{\prime}$ is in $(l, \lambda)$;

(3) The point sequence $M_{0}, M_{1}, M_{2}, \cdots$ is bounded in $\lambda$, where for each nonnegative integer $n, M_{n}$ is the $n$th row of $M$;

(4) There is a point $y$ of $\lambda$ such that $\left|M_{j k}\right| \leqq y_{k}$ whenever each of $j$ and $k$ is a nonnegative integer.

Corollary 5. (See Ch. Rao [4], Th. 2, [5], Lemma to Th. IV, and Tonne [19], Th. 1) Suppose that $0 \leqq r<\infty$ and $M$ is an infinite matrix. The following are equivalent:

(1) $M$ is in $\left(\mathscr{S}_{r}, m\right)$;

(2) There are numbers $t$ and $s$ such that $0<s<1 / r$ and $\left|M_{j k}\right| \leqq t s^{k}$ whenever each of $j$ and $k$ is a nonnegative integer;

(3) $M^{\prime}$ is in $\left(l, \mathscr{g}_{1 / r}\right)$.

For perfect spaces, Theorem 2 can be stated in terms of infinite matrices:

THEOREM 6. Suppose $\lambda$ is a perfect simple space and $M$ is an infinite matrix. The following are equivalent:

(1) $M$ is in $\left(\lambda^{*}, c\right)$;

(2) The point sequence $M_{0}, M_{1}, M_{2}, \cdots$ converges weakly in $\lambda$;

(3) Each column of $M$ is in c, and there is a point $y$ of $\lambda$ such that $\left|M_{j k}\right| \leqq y_{k}$ whenever each of $j$ and $k$ is a nonnegative integer.

Corollary 6. (See Ch. Rao [3], Th. III, and Tonne [19], Th. 2) Suppose $0 \leqq r<\infty$ and $M$ is an infinite matrix. The following are equivalent :

(1) $M$ is in $\left(\mathscr{S}_{r}, c\right)$;

(2) Each column of $M$ is in $c$, and there exist numbers $t$ and s such that $0<s<1 / r$ and $\left|M_{j k}\right| \leqq t s^{k}$ whenever each of $j$ and $k$ is a nonnegative integer.

Corollary 7. Suppose $\lambda$ is a perfect simple space and $M$ is an infinite matrix. The following are equivalent:

(1) $M$ is in $\left(\lambda^{*}, c_{0}\right)$; 
(2) The point sequence $M_{0}, M_{1}, M_{2}, \cdots$ converges weakly in $\lambda$ to the point 0 ;

(3) Each column of $M$ is in $c_{0}$, and there is a point $y$ of $\lambda$ such that $\left|M_{j k}\right| \leqq y_{k}$ whenever each of $j$ and $k$ is a nonnegative integer.

THEOREM 7. Suppose $\lambda$ is a normal space, $\mu$ a normal simple space and $M$ an infinite matrix. The following are equivalent:

(1) $M$ is in $(\lambda, \mu)$;

(2) For each point $x$ of $\lambda$, there is a point $y$ of $\mu$ such that

$$
\sum_{k=0}^{\infty}\left|M_{j k} x_{k}\right| \leqq y_{j}
$$

whenever $j$ is a nonnegative integer.

If $\mu$ is perfect and $\lambda$ contains $\varphi$, the following is equivalent to (1) and

(3) $M^{\prime}$ is in $\left(\mu^{*}, \lambda^{*}\right)$.

Proof. (1) $\rightarrow(2)$. Suppose $x$ is a point of $\lambda$. Let $X$ denote the set to which $z$ belongs if and only if $\left|x_{n}\right|=\left|z_{n}\right|$ for every nonnegative integer $n$. Since $X$ is a bounded subset of $\lambda, M(X)$ is a bounded subset of $\mu$. Let $y$ denote a point of $\mu$, every term of which is a nonnegative real number, that dominates $M(X)$. Then

$$
\sum_{k=0}^{\infty}\left|M_{j k} x_{k}\right| \leqq y_{j}
$$

whenever $j$ is a nonnegative integer.

$(2) \rightarrow(1)$. Suppose $x$ is a point of $\lambda$ and $y$ is a point of $\mu$ such that

$$
\sum_{k=0}^{\infty}\left|M_{j k} x_{k}\right| \leqq y_{j}
$$

whenever $j$ is a nonnegative integer. Then, for each nonnegative integer $j,\left|(M x)_{j}\right| \leqq y_{j}$. Since $\mu$ is normal, $M x$ is a point of $\mu$.

The equivalence of statements (1) and (3) under the additional hypothesis is a consequence of Theorem $\mathrm{B}$.

Corollary 8. Suppose $\lambda$ is a perfect simple space and $M$ is an infinite matrix. The following are equivalent:

(1) $M$ is in $\left(\lambda^{*}, l\right)$;

(2) $M^{\prime}$ is in $(m, \lambda)$;

(3) There is a linear subspace $\mu$ of $m$ containing $c_{0}$ such that $M^{\prime}$ is in $(\mu, \lambda)$ 
(4) There is a point $y$ of $\lambda$ such that

$$
\sum_{j=0}^{\infty}\left|M_{j k}\right| \leqq y_{k}
$$

whenever $k$ is a nonnegative integer.

Corollary 9. (See Tonne [19], Th. 3) Suppose $0 \leqq r<\infty$ and $M$ is an infinite matrix. The following are equivalent:

(1) $M$ is in $\left(\mathscr{S}_{r}, l\right)$;

(2) There exist numbers $t$ and $s$ such that $0<s<1 / r$ and

$$
\sum_{j=0}^{\infty}\left|M_{j k}\right| \leqq t s^{k}
$$

for every nonnegative integer $k$.

Corollary 10. (See Brown [2], Th. 1) Suppose $0 \leqq q<\infty$ and $0 \leqq r<\infty$ and $M$ is an infinite matrix. The following are equivalent:

(1) $M$ is in $\left(\mathscr{S}_{q}, \mathscr{S}_{r}\right)$;

(2) For each number $s$ such that $0<s<1 / r$, there exist numbers $t$ and $p$ such that $0<p<1 / q$ and

$$
\sum_{j=0}^{\infty}\left|M_{j k}\right| s^{j} \leqq t p^{k}
$$

for every nonnegative integer $k$.

If each of $X$ and $Y$ is a set of complex sequences then $X \cdot Y$ denotes the set of all complex sequences of the form $x_{0} y_{0}, x_{1} y_{1}, x_{2} y_{2}, \cdots$ where $x$ is an element of $X$ and $y$ is an element of $Y$. A sequence space $\lambda$ is said to be nuclear with respect to $\lambda^{*}$ whenever $\lambda^{*} \subseteq l \cdot \lambda^{*}$. It is easy to see that $\mathscr{S}_{r}$ and $\mathscr{J}_{1 / r}$ are nuclear with respect to each other $(0 \leqq r<\infty)$.

When $\mu$ is perfect and nuclear with respect to $\mu^{*}$, the following stronger version of Theorem 7 holds:

THEOREM 8. Suppose $\lambda$ is a normal space containing $\varphi$ whose dual is simple, $\mu$ a perfect space that is nuclear with respect to $\mu^{*}$, and $M$ an infinite matrix. The following are equivalent:

(1) $M$ is in $(\lambda, \mu)$;

(2) For each point $x$ of $\mu^{*}$, there is a point $y$ of $\lambda^{*}$ such that $\left|M_{j k} x_{j}\right| \leqq y_{k}$ whenever each of $j$ and $k$ is a nonnegative integer;

(3) $M^{\prime}$ is in $\left(\mu^{*}, \lambda^{*}\right)$. 
Proof. (1) $\rightarrow(2)$. By Theorem B, $M^{\prime}$ is in $\left(\mu^{*}, \lambda^{*}\right)$. By Theorem 7 , if $x$ is a point of $\mu^{*}$, there is a point $y$ of $\lambda^{*}$ such that

$$
\sum_{k=0}^{\infty}\left|M_{j k}^{\prime} x_{k}\right| \leqq y_{j}
$$

whenever $j$ is a nonnegative integer. It follows that $\left|M_{j k} x_{j}\right| \leqq y_{k}$ whenever each of $j$ and $k$ is a nonnegative integer.

$(2) \rightarrow(1)$. Suppose $u$ is a point of $\lambda$ and $v$ is a point of $\mu^{*}$. There exist a point $a$ of $l$, a point $x$ of $\mu^{*}$ and a point $y$ of $\lambda^{*}$ such that $\left|v_{j}\right| \leqq\left|a_{j} x_{j}\right|$ and $\left|M_{j k} x_{j}\right| \leqq\left|y_{k}\right|$ whenever each of $j$ and $k$ is a nonnegative integer. Consequently,

$$
\begin{aligned}
\sum_{j=0}^{\infty}\left|(M u)_{j} v_{j}\right| & \leqq \sum_{j=0}^{\infty} \sum_{k=0}^{\infty}\left|M_{j k}\right|\left|u_{k}\right|\left|v_{k}\right| \\
& \leqq \sum_{j=0}^{\infty}\left|a_{j}\right| \sum_{k=0}^{\infty}\left|M_{j k}\right|\left|x_{j}\right|\left|u_{k}\right| \\
& \leqq \sum_{j=0}^{\infty}\left|a_{j}\right| \sum_{k=0}^{\infty}\left|u_{k} y_{k}\right| .
\end{aligned}
$$

Therefore, $M u$ is a point of $\mu$. It follows that $M$ is in $(\lambda, \mu)$.

$(1) \leftrightarrow(3)$. The equivalence of statements (1) and (3) is a consequence of Theorem B.

Corollary 11. (See Ch. Rao [3], Th. VII, and Fricke and Powell [7], Th. 3) Suppose $0 \leq q<\infty$ and $0 \leqq r<\infty$ and $M$ is an infinite matrix. The following are equivalent:

(1) $M$ is in $\left(\mathscr{S}_{q}, \mathscr{S}_{r}\right)$;

(2) For each number $s$ such that $0<s<1 / r$, there exist numbers $t$ and $p$ such that $0<p<1 / q$ and $\left|M_{j k}\right| s^{j} \leqq t p^{k}$ whenever each of $j$ and $k$ is a nonnegative integer.

Many other classes of matrices could be characterized, such as $\left(\mathscr{S}_{r}, \mathscr{J}_{s}\right),\left(\mathscr{F}_{s}, \mathscr{S}_{r}\right),\left(\mathscr{S}_{r}, c_{0}\right),\left(m, \mathscr{S}_{r}\right),\left(l, \mathscr{S}_{r}\right)$ and $\left(c, \mathscr{S}_{r}\right)$. (See [3], [4], [5], [9] and [19].) In addition, there are similar theorems involving $\mathscr{F}_{r}$ (See [3], [4], [5], [8] and [15].)

Finally, there are other familiar simple spaces, including $m, \omega, \varphi$ and many of the spaces studied by Maddox and his students (cf. [13], [14] and [16]). Similar theorems could be stated for them.

The author wishes to acknowledge several useful comments and suggestions of the referee. In particular, the formulation and proof of Theorem 2 and Corollary 2 given here was essentially suggested by the referee. 


\section{REFERENCES}

1. G. Bennett, Some inclusion theorems for sequence spaces, Pacific J. Math., 46 (1973), 17-30.

2. H. I. Brown, Entire methods of summation, Compositio Math., 21 (1969), 35-42.

3. K. Chandrasekhara Rao, Matrix transformations of some sequence spaces, Pacific J. Math., 31 (1969), 171-174.

4. - Matrix transformations of some sequence spaces, II, Glasgow Math. J., 11 (1970), 162-166.

5. __ Matrix transformations of some sequence spaces, III, Boll. Un. Mat. Ital. (4), 5 (1972),

$7-13$.

6. R. G. Cooke, Linear Operators, Macmillan, 1953.

7. G. Fricke and R. E. Powell, A theorem on entire methods of summation, Compositio Math., 22 (1970), 253-259.

8. I. Heller, Contributions to the theory of divergent series, Pacific J. Math., 2 (1951), 153-177.

9. Roy T. Jacob, Jr., Some matrix transformations on analytic sequence spaces, Pacific J. Math., 59 (1975), 487-491.

10. Gottfried Köthe, Neubegründung der Theorie der Vollkommenen Räume, Math. Nachr., 4 (1951), 70-80.

11. Gottfried Köthe, Topological Vector Spaces I, Springer-Verlag, 1969.

12. G. Köthe and $\mathrm{O}$. Toeplitz, Lineare Räume mit unendlichen Koordinaten und Ringe unendlicher Matrizen, J. Reine Angew. Math., 171 (1934), 193-226.

13. C. G. Lascarides, A study of certain sequence spaces of Maddox and a generalization of a theorem of Iyer, Pacific J. Math., 38 (1971), 487-500.

14. C. G. Lascarides and I. J. Maddox, Matrix transformations between some classes of sequences, Proc. Camb. Phil. Soc., 68 (1970), 99-104.

15. M. S. Macphail, Some theorems on absolute summability, Canad. J. Math., 3 (1951), 386-390.

16. I. J. Maddox, Operators on the generalized entire sequences, Proc. Cambridge Phil. Soc., 71 (1972), 491-494.

17. A. K. Snyder and A. Wilansky, Inclusion theorems and semiconservative FK spaces, Rocky Mountain J. Math., 2 (1972), 595-603.

18. Otto Toeplitz, Die linearen vollkommenen Räume der Funktionentheorie, Comment. Math: Helv., 23 (1949), 222-242.

19. Philip C. Tonne, Matrix transformations on the power-series convergent on the unit disc, J. London Math. Soc. (2), 4 (1972), 667-670.

Received February 10, 1976 and in revised form March 21, 1977.

UNIVERSity OF New ORLEANS

Current address: UNIVERSITY OF DALLAS

IRVING, TX 75061 




\section{Pacific Journal of Mathematics \\ Vol. 70, No. $1 \quad$ September, 1977}

William H. Barker, Noether's theorem for plane domains with hyperelliptic

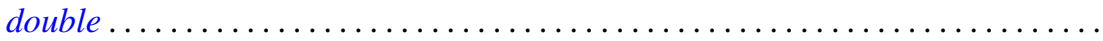

Michael James Beeson, Non-continuous dependence of surfaces of least area on the boundary curve ...................................... 11

Horst Behncke, Functions acting in weighted Orlicz algebras . . . . . . . . . . . . 19

Howard Edwin Bell, A commutativity study for periodic rings . . . . . . . . . . . 29

Peter Botta and Stephen J. Pierce, The preservers of any orthogonal group ....... 37

Douglas S. Bridges, The constructive Radon-Nikodým theorem ............. 51

James Dennis Brom, The theory of almost periodic functions in constructive

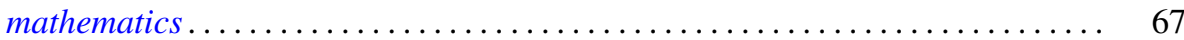

N. Burgoyne and C. Williamson, Semi-simple classes in Chevalley type groups ....

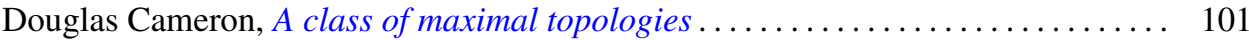

L. Carlitz, Enumeration of doubly up-down permutations . . . . . . . . . . . . . . 105

Paul Robert Chernoff, The quantum n-body problem and a theorem of

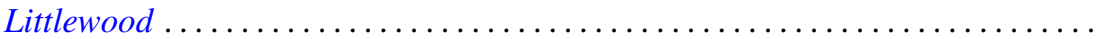

Jo-Ann Deborah Cohen, Locally bounded topologies on $F(X) \ldots \ldots \ldots \ldots \ldots \ldots$

Heinz Otto Cordes and Robert Colman McOwen, Remarks on singular elliptic

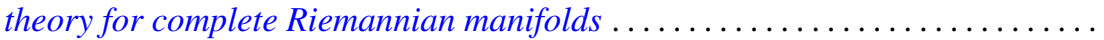

Micheal Neal Dyer, Correction to: "Rational homology and Whitehead

products"

Robert Fernholz, Factorization of Radonifying transformations

Lawrence Arthur Fialkow, A note on quasisimilarity. II ...... . .

Harvey Charles Greenwald, Lipschitz spaces of distributions on the surface of unit

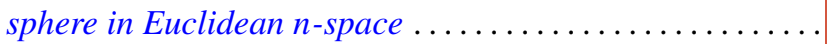

Albrecht Irle, On the measurability of conditional expectations

Tom (Roy Thomas Jr.) Jacob, Matrix transformations involving simple sequence

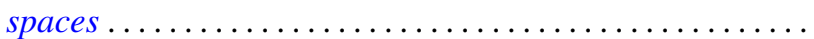

A. Katsaras, Continuous linear maps positive on increasing continuous

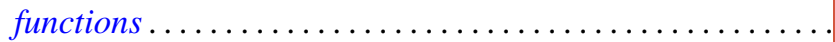

Kenneth Kunen and Judith Roitman, Attaining the spread at cardinals of cofinality

Lawrence Louis Larmore and Robert David Rigdon, Enumerating normal bundles

of immersions and embeddings of projective spaces ...... . .

Ch. G. Philos and V. A. Staïkos, Asymptotic properties of nonoscillatory solutions of differential equations with deviating argument .

Peter Michael Rosenthal and Ahmed Ramzy Sourour, On operator algebras containing cyclic Boolean algebras...

Polychronis Strantzalos, Strikt fast gleichgradig-stetige und eigentliche

Aktionen ...

Glenn Francis Webb, Exponential representation of solutions to an abstract

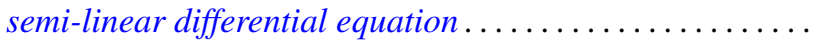

